\title{
UAV Quadrotor Fault Detection and Isolation Using Artificial Neural Network and Hammerstein-Wiener Model
}

\author{
Ahmed Youssef OUADINE ${ }^{1,2 *}$, Mostafa MJAHED ${ }^{1}$, Hassan AYAD ${ }^{2}$, Abdeljalil EL KARI ${ }^{2}$ \\ ${ }^{1}$ Mathematics and Systems Dept; Ecole Royale de l'Air, Marrakech, 40000, Morocco \\ a.y.ouadine@gmail.com (*Corresponding author),mjahed.mostafa@gmail.com \\ ${ }^{2}$ LSEET, Department of Applied Physics, Faculty of Science and Technology Gueliz, \\ Marrakech, 40000, Morocco \\ ayad.ha@gmail.com, jalil.elkari@gmail.com
}

\begin{abstract}
In this paper, a sensor fault diagnosis system is proposed for an aerial vehicle (UAV) quadrotor. Given the nonlinearity of this system and the inaccuracies of modeling, adapted tools have been adopted to ensure control and diagnosis. After synthesizing control laws required for quadrotor control using the Sliding Mode Control method, a HammersteinWiener model has been developed. The goal is to estimate the states of the quadrotor system and build a set of residuals to detect sensor faults. The advantage of this solution is that it does not require prior knowledge of the model and can be easily generalized to other types of vehicles. Then, to ensure decision making for fault isolation, the neural network has been combined with the diagnostic system. With judicious choice of configuration, it can efficiently classify defects from residuals. Finally, typical sensor failures have been injected during simulations. The results of the diagnosis have been very satisfactory. The model has been validated by the test data.
\end{abstract}

Keywords: Quadrotor, Fault detection, Artificial Neural Network, Hammerstein-Wiener.

\section{Introduction}

Over the last decade, many potential applications and technological advances have generated increasing interest in Unmanned Aerial Vehicle (UAV). This interest is due their wide range of civilian and military applications, to the simplicity of their construction and also to their vertical ability to take-off and landing capability. In addition to their cost-effective commercial applications, they have proven their capabilities in the field of exploration of dangerous environments such as burning forests, radioactive zones, hostile area requiring a military reconnaissance mission etc. (Hößler \& Landgraf, 2014).

The new challenging conditions faced by UAVs have shown how critical safety and reliability are to achieving mission objectives and avoiding unnecessary losses. To meet this request, particular importance has been given to the diagnosis of actuator and sensor faults, which lead to undesirable behavior, or even instability of UAVs. Thus, much research has been devoted to their control and diagnosis (Zhang et al., 2013).

To achieve this goal different strategies have been followed in the literature. In most of the cases, when using model-based techniques, the simplest and most practical solution is to linearize the system around a suitable operating point. This solution is especially suitable for UAVs operating most of the time close to the considered conditions. However, this approach does not give satisfactory results for highly nonlinear systems with a wide dynamic operating range. Other methods have been developed directly dealing with models of nonlinear dynamic systems (Sjöberg et al., 1995).

In the field of system controls, PID controller can be mentioned (El Hamidi et al., 2019). The control based on the sliding mode is proposed in (Nguyen \& Hong, 2018). The backstepping approach is applied in (Madani \& Benallegue, 2006) and the hybrid control combining the sliding mode and the backstepping is presented in (Smaoui, Brun, \& Thomasset 2006). Other strategies which hybridize backstepping, sliding mode and fuzzy logic are developed in (Ibraheem, Bahgat \& Motelb, 2000).

As regards the diagnosis of actuator and sensor faults; different methods have been studied. Modelbased methods have been adopted to generate residuals and to detect faults in systems. Luenberger observers have been used for the detection of faults in sensors and actuators (Heredia et al. 2008) and the Kalman filters have been used for checking fault tolerance (Qi et al., 2014).

Other model generating residuals are correctly aimed at ill-defined, complex, and nonlinear processes. There can be mentioned Neural networks, Hammerstein, Wiener, Volterra and Kolmogorov-Gabor models (Isermann, 2005). This approach consists in building a model by identifying the parameters of the system. The diagnosis is then carried out, by monitoring the difference between the numerical values measured 
on the system and their values predicted by the outputs of the constructed model.

The residual is then evaluated for decision making regarding the occurrence of a fault. This task requires good expertise and must be performed in real time.

Several paths have been explored by researchers in the field of fault monitoring. First the statistical methods have been used (Mjahed \& Proriol, 1989). Then, with the improved power of calculators, artificial intelligence techniques such as neural networks, genetic algorithms, fuzzy logic and other techniques have given more satisfaction (Chen et al., 2018).

The contribution and the originality of this article is that it combines the Hammerstein-Wiener model distinguished for its capacity to take into account the nonlinearities of the processes with the Neural Networks in order to ensure a continuous and effective diagnosis.

This article is organized as follows: Section 2 is devoted to the study of the dynamics of the quadrotor and its modeling. The Sliding Method has been used for the quadrotor control. The control laws have been synthesized from the results of UAV modeling. This approach is suitable for the nonlinear model that has been studied.

Section 3 describes the system adopted for fault diagnosis. First, the basic concept of system identification and residual generation by the Hammerstein-Wiener model will be detailed, and then the artificial neurons will be presented as an efficient and reliable method for diagnosing faults. The application of the methods mentioned above on the quadrotor model and the discussion of the simulation results will be detailed and discussed in section 4. Finally, a general conclusion of the work is provided in section 5 .

\section{Quadrotor Modeling and Sliding Mode Control}

This section is devoted to dynamic modelling of the quadrotor; this system and its general principle of flight are firstly described. Then, the dynamic model and the model state space representation are highlighted. Finally, the theoretical foundations of the sliding mode control are detailed before the presentation of the results of its application on the quadrotor.

\subsection{Quadrotor Modeling}

In this subsection, the mathematical model of the quadrotor is briefly explained. A quadrotor is a four-rotor aerial mobile robot generating 4 propeller forces $F_{1}, F_{2}, F_{3}$, and $F_{4}$ (Figure 1). It is necessary that two pairs of propellers (1-3) and (2-4) rotate in opposite directions to balance the moments and produce the required yaw motion. The operation of a quadrotor is quite particular. By varying the power of the motors, it can be moved up/down, tilted left/right (roll) or forward/backward (pitch) or turned on itself (yaw). The quadrotor has six degrees of freedom, three rotational motions, and three translational motions; these six degrees must be controlled with only four triggers; therefore it is an under-actuated system (the number of entries less than the number of outputs) (Mahony, Kumar \& Corke, 2012).

To evaluate the mathematical model of the quadrotor two reference frames are used: the earthfixed inertial reference frame ' $E$ ' and the bodyfixed reference frame ' $B$ ' The absolute position of the quadrotor is described by its coordinates $\mathrm{x}, \mathrm{y}$ and $\mathrm{z}$ and the attitude by the angles of Euler $\varphi, \theta$ and $\psi$; where $\varphi$ is the roll angle (rotation around the $x$-axis), $\theta$ is the pitch angle (rotation around the $y$-axis) and $\psi$ is the yaw angle (rotation around the $z$-axis).

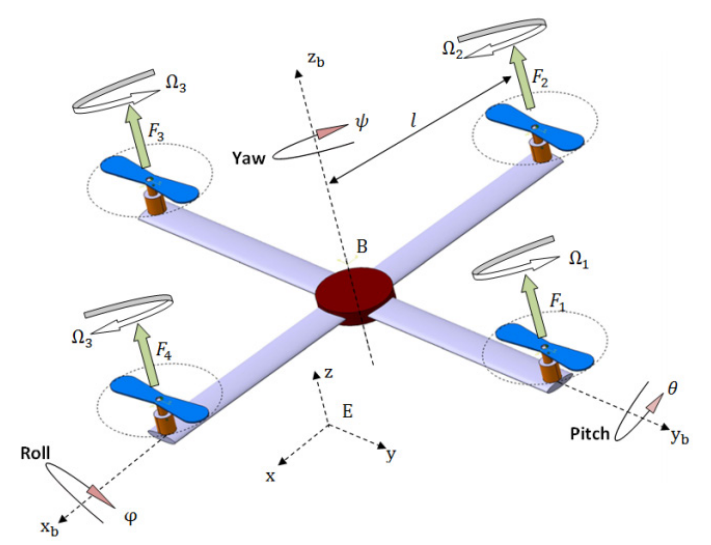

Figure 1. Quadrotor configuration

The torque $\tau$ and the thrust force $F_{i}$ produced by the $i^{\text {th }}$ motor are related to the rotational speed as follows:

$$
\begin{gathered}
F_{i}=b \Omega_{\mathrm{i}}^{2} \\
\tau_{i}=d \Omega_{i}^{2}
\end{gathered}
$$

where $\Omega_{\mathrm{i}}$ is the rotation speed of the $\mathrm{i}^{\text {th }}$ motor and $b$, $d$ are the thrust and drag coefficients, respectively. 
Assume that the control variables can be described as:

$$
\begin{aligned}
& U_{Z}=F_{1}+F_{2}+F_{3}+F_{4} \\
& U_{\varphi}=l\left(F_{3}-F_{4}\right) \\
& U_{\theta}=l\left(F_{1}-F_{2}\right) \\
& U_{\psi}=\tau_{1}+\tau_{2}-\tau_{3}-\tau_{4}
\end{aligned}
$$

where $l$ is the distance between the center of the quadrotor and each rotation axis of the propellers.

The insertion of equations (1) into equations (2) yields:

$$
\begin{aligned}
& U_{z}=\Omega_{1}^{2}+\Omega_{2}^{2}+\Omega_{3}^{2}+\Omega_{4}^{2} \\
& U_{\varphi}=l b\left(\Omega_{3}^{2}-\Omega_{4}^{2}\right) \\
& U_{\theta}=l b\left(\Omega_{1}^{2}-\Omega_{2}^{2}\right) \\
& U_{\psi}=\Omega_{1}^{2}+\Omega_{2}^{2}-\Omega_{3}^{2}-\Omega_{4}^{2}
\end{aligned}
$$

Using the Newton-Euler formalism the dynamic equations are written in the following form (Siti et al., 2019):

$$
\begin{aligned}
& \dot{Z}=\frac{\cos (\varphi) \cos (\theta)}{m} U_{Z}-\frac{K_{Z}}{m} \dot{Z}-g \\
& \dot{\varphi}=\frac{1}{I_{x}} U_{\varphi}+\frac{I_{y}-I_{z}}{I_{x}} \dot{\theta} \dot{\psi}-\frac{J_{T}}{I_{x}} \dot{\theta} \Omega-\frac{K_{\varphi}}{I_{x}} \dot{\varphi} \\
& \dot{\theta}=\frac{1}{I_{x}} U_{\varphi}+\frac{I_{y}-I_{z}}{I_{v}} \dot{\varphi} \dot{\psi}-\frac{J_{T}}{I_{x}} \dot{\varphi} \Omega-\frac{K_{\theta}}{I_{y}} \dot{\theta} \\
& \psi=\frac{1}{I_{z}} U_{\psi}+\frac{I_{x}-I_{y}}{I_{z}} \dot{\varphi} \dot{\theta}-\frac{K_{\psi}}{I_{z}} \dot{\psi}
\end{aligned}
$$

where $\Omega=\Omega_{3}+\Omega_{4}-\Omega_{1}-\Omega_{2}$

The other parameters are defined as follows:

- $I_{x}, I_{y} I_{z}$ : the moments of inertia along the $x, y$, $z$ directions;

- $\quad K_{\varphi^{\prime}} K_{\theta}, K_{\psi}$ : the drag coefficients;

- $\quad J_{T}$ : the moment of inertia of each motor.

At low speed, a simplified nonlinear model of equations (4) can be obtained by neglecting the terms of drag and the gyroscopic effects:

$$
\begin{aligned}
& \dot{Z}=\frac{\cos (\varphi) \cos (\theta)}{m} U_{z}-g \\
& \dot{\varphi}=\frac{1}{I_{x}} U_{\varphi}+\frac{I_{y}-I_{z}}{I_{x}} \dot{\theta} \dot{\psi} \\
& \dot{\theta}=\frac{1}{I_{x}} U_{\varphi}+\frac{I_{y}-I_{z}}{I_{y}} \dot{\varphi} \dot{\psi} \\
& \dot{\psi}=\frac{1}{I_{z}} U_{\psi}+\frac{I_{x}-I_{y}}{I_{z}} \dot{\varphi} \dot{\theta}
\end{aligned}
$$

The parameters of the quadrotor model which are used in this study and which are identified from a real case proposed by (Cen et al., 2013) are shown below.

$$
\begin{array}{ll}
m=1 \mathrm{~kg} & J_{T}=104 \times 10^{-6} \mathrm{~N} . \mathrm{m} / \mathrm{rad} / \mathrm{s}^{2} \\
I_{x}=8,1 \times 10^{-3} \mathrm{~N} . \mathrm{m} / \mathrm{rad} / \mathrm{s}^{2} & I_{y}=8,1 \times 10^{-3} \mathrm{~N} . \mathrm{m} / \mathrm{rad} / \mathrm{s}^{2} \\
I_{z}=14,2 \times 10^{-3} \mathrm{~N} . \mathrm{m} / \mathrm{rad} / \mathrm{s}^{2} &
\end{array}
$$

\subsection{Sliding Mode Controller}

With the complexity of the new systems, conventional controllers become powerless and often give less effective results. To overcome this problem, research tends to favor robust nonlinear controls that yield acceptable results. These techniques include Sliding Mode Control (SMC), which has long been the subject of several research studies (Hung, Gao \& Hung, 1993).

The SMC is used to control nonlinear processes subject to external disturbances and large uncertainties related to modeling. The SMC is a nonlinear control that relies on the switching functions of the state variables used to create a sliding surface. The goal is to force the dynamics of the system to follow the equation defined by this surface and to keep it on this surface. The system is then insensitive to external and parametric disturbances as long as the conditions of the sliding mode are guaranteed. In this subsection some basic notions of the concepts of sliding modes theory will be given.

The SMC of the single input, single output (SISO) system expressed by equations (6) will be studied. This control system will be applied on quadcopter modeled by Equations (5).

$$
\begin{aligned}
& \dot{x}_{1}=x_{2} \\
& x_{2}=g\left(x_{1}, x_{2}, t\right) \cdot u+f\left(x_{1}, x_{2}, t\right) \\
& y=x_{1}
\end{aligned}
$$


where:

- $\quad x_{1}, x_{2}:$ are the state variables;

- $\quad y$ is the controlled outputs $(z, \varphi, \theta$ or $\psi)$;

- $\quad u$ is the controlled input $\left(U_{Z}, U_{\varphi}, U_{\theta}\right.$ or $\left.U_{\psi}\right)$;

- $g\left(x_{1}, x_{2}, t\right):$ is a nonlinear function;

- $f\left(x_{1}, x_{2}, t\right)$ : is the term of perturbation, which may include a dry and viscous friction in addition to any further resistance. It is supposed to be bounded by Equation (7).

$$
\left|f\left(x_{1}, x_{2}, t\right)\right|<|L|
$$

The objective is to design a sliding mode control $u\left(x_{1}, x_{2}, t\right)$ allowing the output to asymptotically follow the input reference $y_{r}$.

Let $e$ be the tracking error (Equation (8))

$$
e(\mathrm{t})=\mathrm{y}(\mathrm{t})-\mathrm{y}_{\mathrm{r}}(\mathrm{t})
$$

The control low $u$ must lead this error to zero: $\left(\lim _{t \rightarrow \infty}(e(t))=0\right)$.

Let $\sigma$ be the Sliding Variable expressed as a function of $e$ and $\dot{e}$ (Equation (9))

$$
\sigma(e, \dot{e})=\dot{e}+\lambda e \quad \lambda>0
$$

In order to ensure the asymptotic convergence of the variables $x_{1}$ and $x_{2}$ the variable $\sigma$ has to be driven to zero in finite time by means of the control $u$, although the presence of the bounded perturbation $f$.

By using the Lyapunov function techniques to $\sigma$-dynamics, this result can be achieved. From Equations (8) and (9) one obtains:

$$
\dot{\sigma}=y_{r}+\dot{y}_{r}-f(y, \dot{y}, t)-\lambda \dot{y}-g \cdot u
$$

The Lyapunov function for the $\sigma$ -dynamics is expressed by Equation (11):

$$
V=\frac{1}{2} \sigma^{2}
$$

The following conditions must be satisfied to ensure the asymptotic stability of Equation (10) around the point of equilibrium $(\sigma=0)$ :

$$
\dot{V}<0, \sigma \neq 0
$$

$$
\lim _{|\sigma| \rightarrow \infty} V=\infty
$$

It can be easily deduced that condition $(12 b)$ is satisfied. With regard to condition (12a) which ensures convergence and stability, it can be modified as follows (Equation (13)):

$\dot{V}(t)<-\alpha V^{1 / 2}, \alpha>0$

After the separation of the variables and the integration on the time interval $0<\tau<t$ one obtains:

$$
V^{1 / 2}(t) \leq-\frac{1}{2} \alpha t+V^{1 / 2}(0)
$$

Consequently, $V(t)$ reaches zero in a finite time $t_{r}$ that is bounded by (Equation (15)).

$$
\mathrm{t}_{\mathrm{r}} \leq \frac{2 \mathrm{~V}^{1 / 2}(0)}{\mathrm{a}}
$$

The control $u$ that satisfies Equation (13) is calculated. This control will drive the variable $\sigma$ to zero in a finite time and then keep it at that value.

The derivative of $V$ is computed as:

$$
\dot{V}=\sigma \sigma^{\ddot{y}}=\sigma\left(\ddot{y}_{r}+\dot{y}_{r}-f(y, \dot{y}, t)-\lambda \dot{y}-g . u\right)
$$

Let be:

$$
\phi=y_{r}+\dot{y}_{r}-f(y, \dot{y}, t)-\lambda \dot{y}
$$

$y_{r}, \dot{y}_{r}:$ are the known input references.

The cumulative disturbance $\phi(y, \dot{y}, t)$ is assumed to be bounded $(|\phi(y, \dot{y}, t)|<D)$

Assuming:

$$
u=\frac{1}{g} \rho \operatorname{sign}(\sigma)
$$

where $\rho$ is the control gain $(\rho>0)$ and 'sign' is a function expressed as follows:

$$
\operatorname{sign}(x)=\left\{\begin{array}{l}
1 \text { if } x>0 \\
-1 \text { if } x<0,
\end{array}, \operatorname{sign}(0) \in[-1,1]\right.
$$

Substituting it into Equations (16) and (17) one obtains:

$$
\dot{V}=\sigma(\phi(y, \dot{y}, t)-\rho \operatorname{sign}(\sigma))
$$


Then it yields:

$\dot{V}=\sigma \phi(y, \dot{y}, t)-\rho \sigma \operatorname{sign}(\sigma)=\sigma \phi(y, \dot{y}, t)-\rho|\sigma|$

It can be deduced:

$$
\sigma \phi(y, \dot{y}, t)-\rho|\sigma|<|\sigma|(D-\rho)
$$

Taking into account Equation (11) and condition (12a) one obtains:

$$
\dot{V}<-\alpha V^{1 / 2}=\frac{-\alpha}{\sqrt{2}}|\sigma|, \alpha>0
$$

The control gain $\rho$ is computed as:

$$
\rho=D+\frac{\alpha}{\sqrt{2}}
$$

\subsection{Control Law Synthesis}

In this subsection the synthesis of the control law which will be applied to the quadrotor system modeled by Equations (5) will be carried out. This task will be accomplished by applying the Sliding Mode Control.

Consider Equation (5b) of the yaw angle ' $\varphi$ ':

$$
\varphi=b_{1} U_{1}+a_{1} \dot{\theta} \dot{\psi}
$$

where: $b_{1}=\frac{l}{I_{x}}$ and $a_{1}=\frac{I_{y}-I_{z}}{I_{x}}$

The goal is to design a control law $U$ that will allow the output $\varphi$ to follow the input reference $\varphi_{\text {ref }}$. The tracking error is expressed as follows:

$$
e_{\varphi}=\varphi_{\text {ref }}-\varphi
$$

\section{By applying Equation (18) one will obtain:}

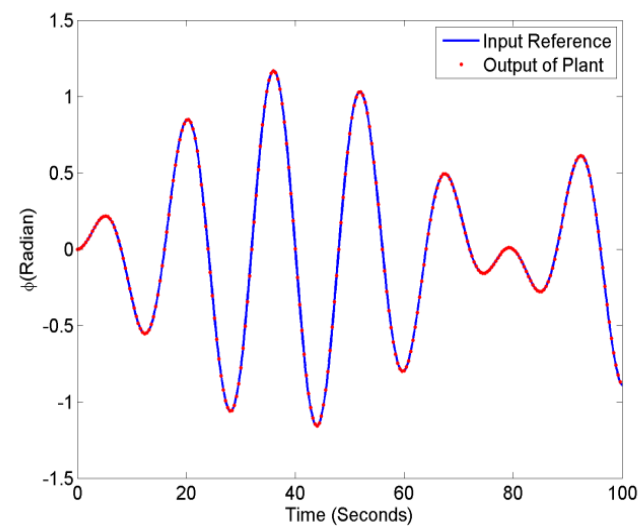

$$
U_{\varphi}=\frac{1}{b_{1}} \rho_{\varphi} \operatorname{sign}\left(\sigma_{\varphi}\right)
$$

Where: $\sigma_{\varphi}=\dot{e}_{\varphi}-\lambda e_{\varphi}$

In a similar way one obtains:

$$
\begin{aligned}
& U_{\theta}=\frac{1}{b_{1}} \rho_{\theta} \operatorname{sign}\left(\sigma_{\theta}\right) \\
& U_{\psi}=\frac{1}{b_{1}} \rho_{\psi} \operatorname{sign}\left(\sigma_{\psi}\right) \\
& U_{z}=\frac{m}{\cos \theta \cos \varphi} \rho_{z} \operatorname{sign}\left(\sigma_{z}\right)
\end{aligned}
$$

with: $0 \leq \theta<\frac{\pi}{2}, 0 \leq \varphi<\frac{\pi}{2}$

The parameters of the Sliding Mode Control are fixed as follows:

$\lambda=1,5$ and $\rho_{z}=\rho_{\varphi}=\rho_{\theta}=\rho_{\psi}=120$.

The simulation has been performed with the following input references:

$$
\begin{aligned}
& Z_{\text {ref }}=\frac{3 \pi}{8} \cos \left(\frac{1}{20} t\right) \sin \left(\frac{\pi}{15} t\right) \\
& \varphi_{\text {ref }}=\frac{3 \pi}{8} \sin \left(\frac{1}{25} t\right) \sin \left(\frac{\pi}{15} t\right) \\
& \theta_{\text {ref }}=\frac{3 \pi}{8} \cos \left(\frac{1}{15} t\right) \sin \left(\frac{\pi}{15} t\right) \\
& \psi_{\text {ref }}=\frac{3 \pi}{8} \sin \left(\frac{1}{15} t\right) \cos \left(\frac{\pi}{15} t\right)
\end{aligned}
$$

Figures (2) shows the simulated input reference and system output for $\varphi$ signal.

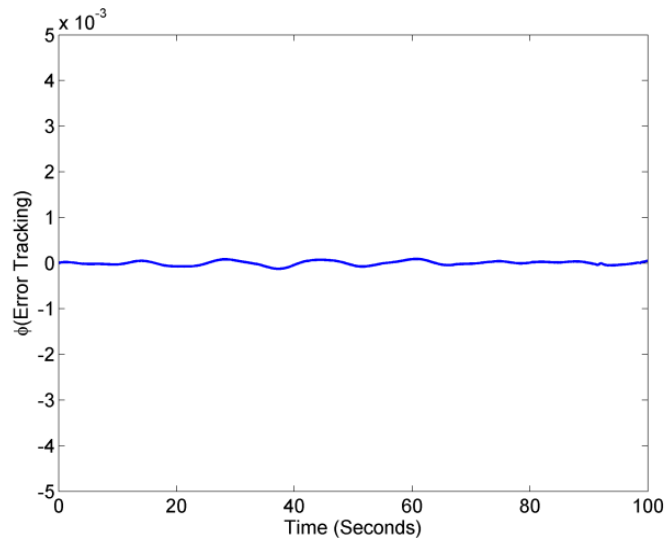

Figure 2. Sliding Mode Control of Signal 


\section{Fault Diagnostic System}

The principle of model-based monitoring is to compare the behavior of the actual system with that of a model that represents normal operative conditions. The general approach is to compare the actual output of the system to an estimated fault free output of the model. Fault indication signals, called residuals, are thus generated to perform a diagnostic decision test (Equation (27)).

$$
r(t)=y(t)-\hat{y}(t)
$$

Where $y(t)$ is the actual output of the system and $\hat{y}(t)$ is the estimated output.

Residuals are designed to be equal or converge to zero in case there are no defects and deviate considerably from zero if an error occurs. Therefore, the residuals characterize the effects of the faults. Depending on the number of the generated residuals and their design, it is possible to detect and isolate the faults that have occurred.

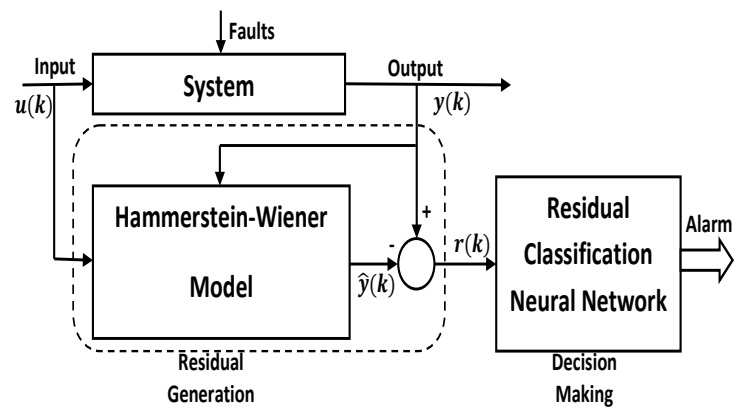

Figure 3. Fault Diagnosis system

Model-based fault diagnosis can therefore be divided into two main stages: residuals generation and decision-making (Figure 3).

To achieve this goal, the Hammerstein-Wiener model has been successively used for residual generation and multilayer artificial neural networks for decision making. Further explanation of these two blocks will be introduced in the following subsections.

\subsection{Hammerstein-Wiener Model}

A relatively simple way to represent the nonlinear behavior of a system relies on the use of structured block models composed of linear dynamic elements and nonlinear static elements. The Hammerstein, Wiener and Hammerstein-Wiener models can be mentioned (Vörös, 2014).
In the Hammerstein model, the input signal passes first through the nonlinear static element to give an intermediate signal which will be processed by the dynamic linear part. The nonlinear element can explain the nonlinearities of the actuators and other effects that can be placed at the input of the system (Wang et al. 2009).

The permutation of the linear and nonlinear elements in the Hammerstein model leads to the Wiener model. This model is well adapted to the characterization of a system whose sensor has a nonlinear behavior (Chen, Lu \& Ding, 2014).

The combination of a Hammerstein model and a Wiener model in series allows the development of a new Hammerstein-Wiener structure. Figure 4 shows the connection between these blocks. The Hammerstein-Wiener models are well suited to the characterization of a system whose actuator and sensor have a nonlinear character. It has been successfully applied to the modeling of several physical processes (Nadimi et al. 2012).

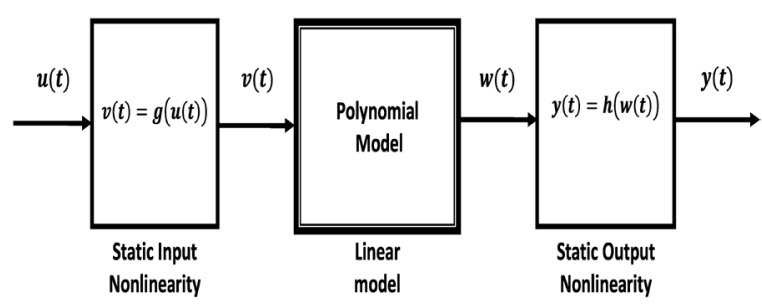

Figure 4. Hammerstein-Wiener Model

Most studies have assumed that the linear element is parametric and can be represented by a known order transfer function, a state space representation or an input-output polynomial model with a known degree of dynamics.

For the polynomial model used in this research, the relationship between the output of the linear subsystem and the input is given in Equation (28):

$$
v(t)=g(u(t))=\frac{B(z)}{A(z)} u(t)
$$

Here ' $z$ ' is the shift operator: $\left[z^{-1} y(t)=y(t-1)\right]$ and $A(z), B(z)$ and $D(z)$ are polynomials.

With regard to nonlinear subsystems, several solutions can be adopted. The piecewise linear function, the sigmoid network, the Wavelet network and the saturation function can be mentioned (Shokrollahi et al., 2018). In the 
present work, the piecewise linear function with 10 break points has been chosen for estimating the two nonlinear Hammerstein-Wiener subsystems.

To allow the identification of the system, it is necessary to have a set of input and output signals of the process. These signals are acquired in normal operation or introduced artificially.

A considerable advantage of the identification methods is that with the input signal and the output signal, several parameters can be estimated, which gives detailed information about the internal behavior of the process. In this study, after configuring the model, the identification data has been artificially generated.

After identifying the parameters of the process model that generates the residuals, it is essential to set up a fault classifier from this data. In the present study, the multilayer neural networks have been chosen as an efficient and reliable method to perform this task. To enable a reliable diagnosis, the training of the network must be done by using the data of all the possible fault situations.

In what follows, a brief overview of the principle of Artificial Neural Networks will be introduced.

\subsection{Artificial Neural Networks}

Artificial Neural Networks (ANN) reproduce the neural network of the biological brain through elementary processing units interconnected by weighted connections. Learning involves modifying these weights to allow these networks to perform specific tasks such as pattern recognition, classification, clustering, and functions approximation (Ouadine et al., 2018).

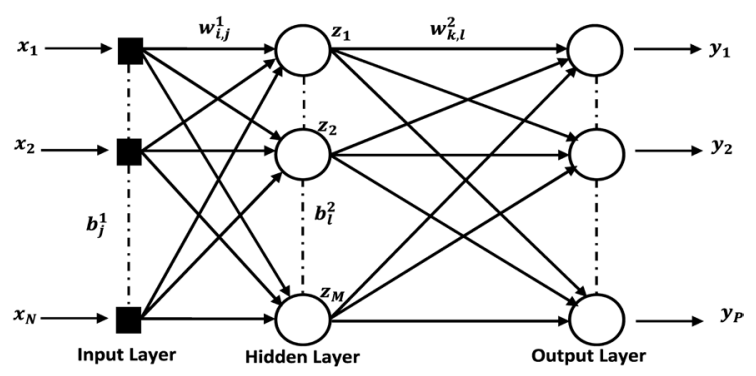

Figure 5. Multilayer Perceptron

Multilayer Perceptron (MLP) is the most popular ANN. It has been used to identify and classify faults into 5 classes. An MLP network includes several layers; each is composed of a set of neurons. The number of neurons in the input and output layer depends on the number of the input variables and number of the desired classes, respectively. Figure 5 illustrates a typical MLP architecture consisting of an input layer, a single hidden layer, and the output layer.

The desired output vector $Y\left(y_{1}, y_{2} \ldots y_{p}\right)$ of the network is calculated from the input vector $X\left(x_{1}, x_{2}, \ldots x_{N}\right)$ and the intermediate vector $z\left(z_{1}, z_{2} \ldots\right.$ $z_{M}$ ) by using equations (28a) and (28b):

$$
\begin{aligned}
& z_{j}=f_{1}\left(\sum_{i=1}^{N} w_{i, j}^{1} x_{i}+b_{j}^{1}\right) \\
& y_{k}=f_{2}\left(\sum_{l=1}^{M} w_{l, k}^{2} z_{l}+b_{k}^{2}\right)
\end{aligned}
$$

where:

- $f_{1}$ and $f_{2}$ are the activation functions such as the sigmoid function, the hyperbolic tangent and the linear function;

$w^{l}$ is the first matrix weight with $w_{i, j}^{1}$ which is the weight between the $j^{\text {th }}$ neuron of the hidden layer and the $i^{\text {th }}$ neuron of the input layer;

- $w^{2}$ is the second matrix weight with $w_{l, k}^{2}$ which is the weight between the 1th neuron of the output layer and the kth neuron of the hidden layer;

$b_{j}^{1}\left(b_{j}^{2}\right)$ is the bias of the $\mathrm{j}^{\text {th }}$ neuron of the first (second) layer

The learning algorithm indicates how to modify the weighting values assigned to the connections between the processing units. It is based on the minimization of a sum of Mean Squared Errors (MSE) between the calculated output and the desired output. The fundamental learning algorithms for multilayer networks are the BackPropagation (BP) (Van Ooyen \& Nienhuis, 1992), Levenberg-Marquardt, Conjugate Gradient and Scaled Conjugate Gradient Back-propagation.

\section{Experimental Results}

In this subsection, the steps to follow in order to create a quadrotor diagnostic system will be introduced. As mentioned earlier, this model is composed of the residual generation and the decision-making blocks. The approach followed to accomplish this work will be presented. 


\subsection{Identification of Hammerstein- Wiener Model}

The first step in building the HammersteinWiener model is to collect identification data from the plant.

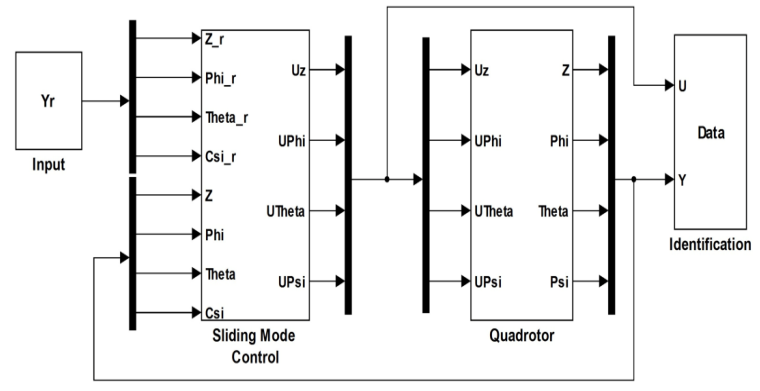

Figure 6. Identification of Model

Figure 6 shows the Simulink block used to collect the 4 control inputs $\left(U_{z}, U_{\varphi}, U_{\theta}, U_{\psi}\right)$ and the 4 outputs $(Z, \varphi, \theta$ and $\psi)$. The duration of each collected signal is 600 seconds with a sampling time of 0.1 second.

The data has been divided into two parts, $50 \%$ for model estimation and $50 \%$ for its validation. As noted in subsection 2.1, the Hammerstein-Wiener model has been used to identify the system. From the generated simulation data, several tests have been performed by using Identification ToolBox of MATLAB, to find the configuration and parameters of the optimal model.

To allow a quantitative comparison the fitness value indicator ' $F i t$ ' has been introduced as performance criteria for both estimation and validation of the data (Equation (29)).

$$
\% \text { Fit }=100 \times\left(1-\sum_{i=1}^{n} \frac{|y(i)-\hat{y}(i)|_{2}}{|y(i)-\bar{y}|_{2}}\right)
$$

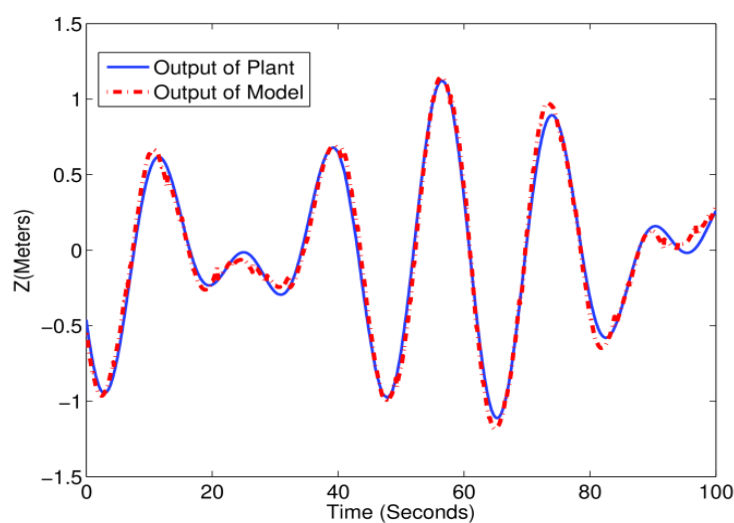

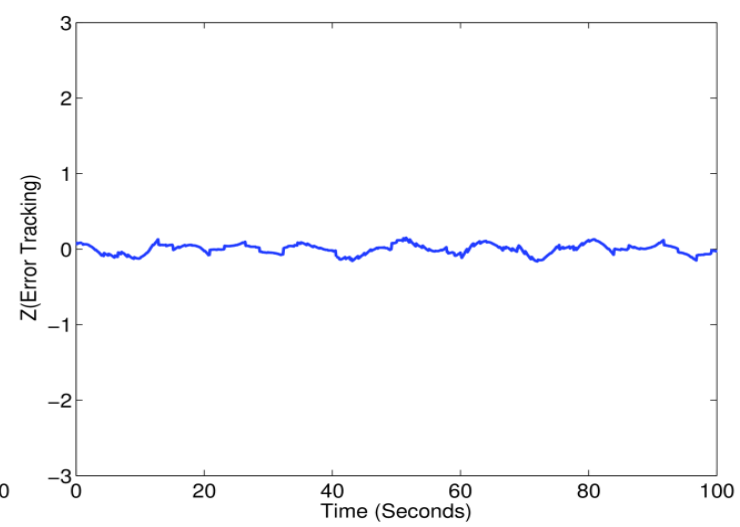

Table 1. Fitness function

\begin{tabular}{ccc}
\hline Output & Estimation & Validation \\
\hline$Z$ & $91,15 \%$ & $90,53 \%$ \\
$\varphi$ & $90,88 \%$ & $90,08 \%$ \\
$\theta$ & $92,61 \%$ & $92,36 \%$ \\
$\psi$ & $95,84 \%$ & $96,27 \%$ \\
\hline
\end{tabular}

It can be seen that the quadrotor system has been well identified by the Hammerstein-Wiener nonlinear model. The fitness value in this model has exceeded the value of $90 \%$. The lowest value of this value is recorded on the roll angle ' $\varphi$ ' output. The differences recorded between the outputs of the process and their estimates by the model remain within the tolerances. This is confirmed by the results of the diagnosis by the classification of residuals which will be seen in subsection 4.2. This model is validated for fault detection and isolation.

\subsection{Residual Generation and Fault Identification}

The present study has been limited to simulating sensor type defects and it has been assumed that there was only one failure at a time. A good diagnosis requires an excellent ability to identify

Figure 7. Estimation of Altitude 
(classify) defects by the ANN. Therefore, their training from a database containing all the possible cases has been carried out: system without faults and with 4 sensor faults $(Z, \varphi, \theta$ and $\psi)$.

Abrupt faults of $0.1 \mathrm{~m}$ amplitude on the altitude sensor ' $Z$ ' and of $0.1 \mathrm{rad}$ amplitude on the other angle sensors have been simulated.

Figure 8 shows how a fault on the quadrotor's ' $\varphi$ ' sensor has been simulated on a Simulink model. The faults simulation allows the generation of the residual signals. The duration of each simulation is 100 seconds with a sampling rate of 0.1 seconds.

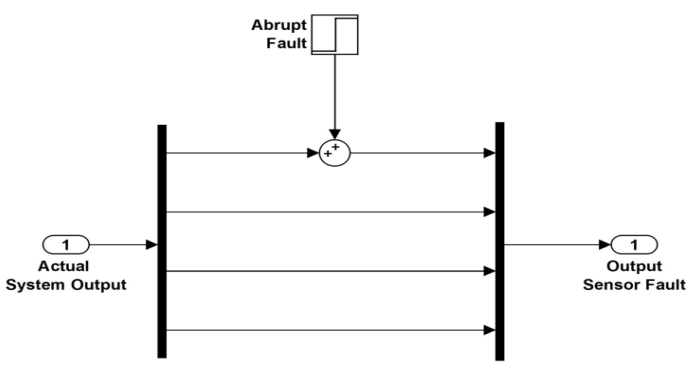

Figure 8. Simulation of abrupt fault (altitude sensor)

Figure 9 shows the values of the generated residual with defects on the sensor ' $\psi$ '.

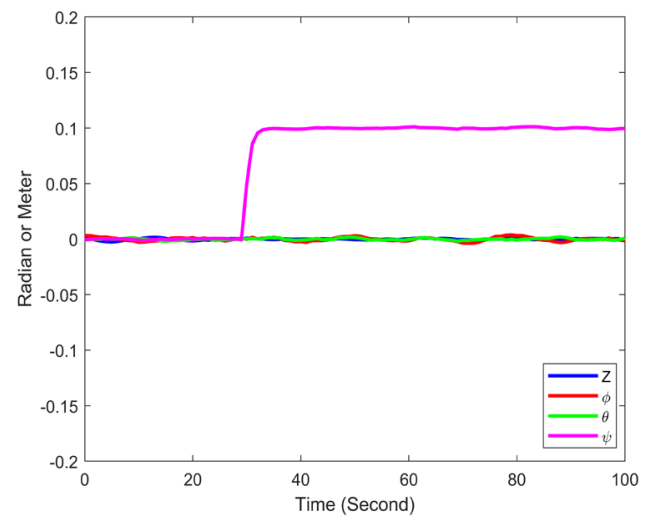

Figure 9. Fault in ' $\psi$ ' Sensor
A training database divided into 5 classes organized in a matrix composed of 5005 rows and 4 columns corresponding to the residual values of $Z, \varphi, \theta$ and $\psi$ has been obtained.

Before starting the training of the ANN, the rows of the data matrix have been normalized to ensure that the data is processed with the same priority. Then database has been divided into three parts: $70 \%$ for the training, $15 \%$ for the validation and $15 \%$ for the test.

The neuron network has been created, trained and implemented using the MATLAB Neural Network Toolbox. The Scaled Conjugate Gradient Backpropagation has been used for training the network and the Hyperbolic Tangent Sigmoid as transfer function.

The ANN, used in this work, is composed of three layers (input, hidden and output). The number of neurons in the input layer is 4 corresponding to the number of residuals generated by the Hammerstein-Wiener model, 10 neurons are in the hidden layer and 5 neurons in the output layer corresponding to the number of classes.

Convergence is reached after 67 periods with a Mean Squared Error MSE $=4.2303 \times 10^{-7}$. The results are very satisfactory with a classification rate of $100 \%$ for training, validation and test data.

Figure 10 shows the Simulink model of the Fault diagnosis system. The simulation has been carried out with the following three blocks: Quadrotor plant, Hammerstein-Wiener model and Artificial Neural Networks block.

In this subsection, the concepts of control and diagnosis seen in the previous sections have been implemented on a quadrotor. The rate of

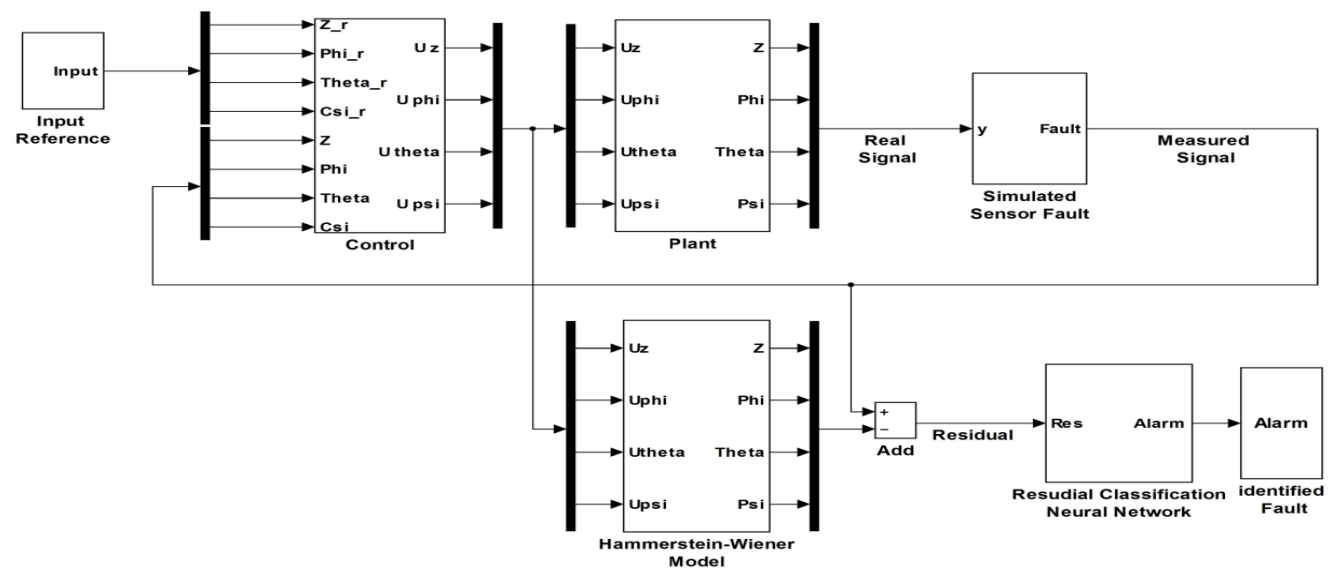

Figure 10. Global diagram of fault detection system 
classification of defects by neural networks from residual data reaches a value of $100 \%$. The results have been validated to diagnose faults in quadrotor sensors.

\subsection{Results Discussion}

In the present work, a diagnostic system for quadrotor sensors has been established. Three steps have been followed.

First, the dynamics of the nonlinear system has been modeled to synthesize the control laws by the sliding mode method. Then, a process model has been estimated to generate the residuals. Finally, the automatic diagnosis has been carried out by the ANN.

The sliding mode control system has been used successfully. Much of the work in the field of quadrotor control relies on linear representations to describe the system. For example, by the PID controller (Pounds, Bersak, \& Dollar, 2012) or Linear Quadratic Regulator (LQR) (Islam \& Okasha, 2019) methods. The advantage of the approach followed in this work compared to these methods is that it takes into account the nonlinearity of the system, the uncertainties of the modeling and the external disturbances.

According to the results obtained in subsection 2.3, the control system has been able to stabilize the quadcopter with the reference inputs (Equations 26).

For fault detection, many techniques are available. The state observer methods (Frank \& Ding, 1997), parity equations (Han, Wang \& Shen, 2017), parameter estimation (Mokhtari \& Benallegue, 2004) are based on linear representations of the system. However, due to linearization approximations, the generated residuals may not provide reliable fault detection information.

To avoid this problem, it has been estimated in this work the nonlinear Hammerstein-Wiener model to generate residuals. This model takes into account the nonlinearities of the actuators and the sensors of the quadrotor. The model has been validated from the simulation results discussed in subsection 4.1.

Finally, to ensure a diagnosis, ANN was used for the classification of defects from the available residuals. This approach is renowned for its efficiency compared to conventional methods (Kourti \& MacGregor, 1995). Indeed, the classification rate is maximum: $(100 \%$ for learning, validation end test data).

\section{Conclusion}

In this work, a system for diagnosing faults in sensors mounted on a quadrotor has been set up. This approach is based on a HammersteinWiener model for generating residuals and on neural networks for classification and automatic diagnosis.

The choice of the residual generation approach based on the Hammerstein-Wiener model is justified by its great ability to take into account the nonlinearities of the sensors and actuators as in the case of the quadrotor. The neural networks have been selected because they have proven their excellent classification performance in the field of fault diagnosis on several applications.

After the synthesis of the control laws by the sliding mode method, the parameters of the Hammerstein-Wiener model have been estimated. Then, to generate residuals, several faults have been simulated on the altitude and attitude sensors. The residuals allow a comparison of the output signals of the real process with the outputs of the process model. They deviate from zero if there is a fault.

For the automatic diagnosis, the learning of neural networks has been carried out from the residual database. The optimal neural network classifying the defects from residuals is composed of a single hidden layer of 10 neurons. Convergence is reached after 67 epochs with a mean square error $M S E=4.2303 \times 10^{-7}$. The diagnostic model has been validated with a classification rate of $100 \%$ for training, validation and test data.

In this work, abrupt type faults have been simulated. This study can be easily generalized to other faults affecting the actuators or the process itself and for the case where several faults occur simultaneously. 


\section{REFERENCES}

Cen, Z. Noura, H. \& Bagus, T. (2013). Robust Fault Diagnosis for Quadrotor UAVs Using Adaptive Thau Observer, Journal of Intelligent \& Robotic Systems, 73(1-4), 573-588. DOI: 10.1007/s10846-013-9921-8

Chen, J., Lu, X. \& Ding, R. (2014). GradientBased Iterative Algorithm for Wiener Systems with Saturation and Dead-Zone Nonlinearities, Journal of Vibration and Control, 20(4), 634-640. DOI: $10.1177 / 1077546312466563$

Chen, R. L., Yang, B., Zio, E. \& Xuefeng, C. (2018). Artificial Intelligence for Fault Diagnosis of Rotating Machinery: A Review, Mechanical Systems and Signal Processing, 108, 33-47.

El Hamidi, K., Mjahed, M., El Kari, A. \& Ayad, H. (2019). Neural Network and Fuzzy-Logic-Based SelfTuning PID Control for Quadcopter Path Tracking, Studies in Informatics and Control, 28(4), 401-412. DOI: $10.24846 /$ v28i4y201904

Frank, P. M. \& Ding, X. (1997). Survey of robust residual generation and evaluation methods in observer-based fault detection systems, Journal of Process Control, 7(6), 403-424. DOI: 10.1016/S09591524(97)00016-4

Han, W., Wang, Z. \& Shen, Y. (2017). Fault Estimation for a Quadrotor Unmanned Aerial Vehicle by Integrating the Parity Space Approach with Recursive Least Squares. In Proceedings of the Institution of Mechanical Engineers, Part G: Journal of Aerospace Engineering, 232(4), (pp. 783-796). DOI: $10.1177 / 0954410017691794$

Heredia, G., Ollero, A., Bejar, M. \& Mahtani, R. (2008). Sensor and Actuator Fault Detection in Small Autonomous Helicopters, Mechatronics, 18(2), 9099. DOI: 10.1016/j.mechatronics.2007.09.007

Hößler, T. \& Landgraf, T. (2014). Automated Traffic Analysis in Aerial Images, Computer Vision and Graphics, 262-269. Springer International Publishing. DOI: 10.1007/978-3-319-11331-9_32

Hung, J. Y., Gao, W. \& Hung, J. C. (1993). Variable Structure Control: A Survey, IEEE Transactions on Industrial Electronics, 40(1), 2-22. DOI: $10.1109 / 41.184817$

Ibraheem, A. A., Bahgat, A. \& Abdel Motelb, M. S. (2000). Fuzzy Logic Sliding Mode Controller for Dc Drive, Current Advances in Mechanical Design and Production VII, 75-83. Pergamon. DOI: 10.1016/ B978-008043711-8/50008-8.

Isermann, R. (2005). Model-Based Fault-Detection and Diagnosis - Status and Applications, Annual Reviews in Control, 29(1), 71-85. DOI: 10.1016/j. arcontrol.2004.12.002
Islam, M. \& Okasha, M. (2019). A Comparative Study of PD, LQR and MPC on Quadrotor Using Quaternion Approach. In 2019 7th International Conference on Mechatronics Engineering (ICOM) (pp. 1-6). DOI: 10.1109/ICOM47790.2019.8952046

Kourti, T. \& MacGregor, J. F. (1995). Process Analysis, Monitoring and Diagnosis, Using Multivariate Projection Methods, Chemometrics and Intelligent Laboratory Systems, 28(1), 3-21. DOI: 10.1016/01697439(95)80036-9

Madani, T. \& Benallegue, A. (2006). Backstepping Control for a Quadrotor Helicopter. In 2006 IEEE/ RSJ International Conference on Intelligent Robots and Systems (pp. 3255-3260). DOI: 10.1109/ IROS.2006. 282433

Mahony, R., Kumar, V. \& Corke, P. (2012). Multirotor Aerial Vehicles: Modeling, Estimation, and Control of Quadrotor, IEEE Robotics \& Automation Magazine, 19(3), 20-32. DOI: 10.1109/MRA.2012. 2206474

Mjahed, M. \& Proriol, J. (1989). Taging Jets and Partons by Discriminant Analysis, Physics Letters $B$, 217(4), 560-562. DOI: 10.1016/0370-2693 (89)90096-8

Mokhtari, A. \& Benallegue, A. (2004). Dynamic Feedback Controller of Euler Angles and Wind Parameters Estimation for a Quadrotor Unmanned Aerial Vehicle. In Proceedings of the IEEE International Conference on Robotics and Automation, ICRA '04, Vol 3 (pp. 2359-2366). DOI: 10.1109/ ROBOT.2004.1307414

Nadimi, E. S., Green, O., Blanes-Vidal, V., Larsen, J. J. \& Christensen, L. P. (2012). Hammerstein-Wiener Model for the Prediction of Temperature Variations inside Silage Stack-Bales Using Wireless Sensor Networks, Biosystems Engineering, 112(3), 236-247. DOI: 10.1016/j.biosystemseng.2012.04.007

Nguyen, N. P. \& Hong, S. K. (2018). Sliding Mode Thau Observer for Actuator Fault Diagnosis of Quadcopter UAVs, Applied Sciences, 8(10), 1893. DOI: $10.3390 / a p p 8101893$

van Ooyen, A. \& Nienhuis, B. (1992). Improving the Convergence of the Back-Propagation Algorithm, Neural Networks, 5(3), 465-471. DOI: 10.1016/08936080(92)90008-7

Ouadine, A. Y., Mjahed, M., Ayad, H. \& El Kari, A. (2018). Aircraft Air Compressor Bearing Diagnosis Using Discriminant Analysis and Cooperative Genetic Algorithm and Neural Network Approaches, Applied Sciences, 8(11), 2243. DOI: 10.3390/app8112243

Pounds, P. E. I., Bersak, D. R. \& Dollar, A. M. (2012). Stability of Small-Scale UAV Helicopters 
and Quadrotors with Added Payload Mass under PID Control, Autonomous Robots, 33(1), 129-142. DOI: 10.1007/s10514-012-9280-5

Qi, X., Qi, J., Theilliol, D., Zhang, Y., Han, J., Song, D. \& Hua, C. (2014). A Review on Fault Diagnosis and Fault Tolerant Control Methods for Single-Rotor Aerial Vehicles, Journal of Intelligent \& Robotic Systems, 73(1-4), 535-55. DOI: 10.1007 /s10846013-9954-z

Shokrollahi, E., Goldenberg, A., Drake, J., Eastwood, K. \& Kang, M. (2018). Application of a Nonlinear Hammerstein-Wiener Estimator in the Development and Control of a Magnetorheological Fluid Haptic Device for Robotic Bone Biopsy, Actuators, 7(4), 83. DOI: $10.3390 /$ act7040083

Siti, I., Mjahed, M., Ayad, H. \& El Kari, A. (2019). New Trajectory Tracking Approach for a Quadcopter Using Genetic Algorithm and Reference Model Methods, Applied Sciences, 9(9), 1780. DOI: 10.3390/ app 9091780

Sjöberg, J., Zhang, Q., Ljung, L., Benveniste, A., Delyon, B., Glorennec, P., Hjalmarsson, H. \& Juditsky, A. (1995). Nonlinear Black-Box Modeling in System Identification: A Unified Overview, Automatica,
31(12), 1691-1724. DOI: 10.1016/0005-1098 (95) 00120-8

Smaoui, M., Brun, X. \& Thomasset, D. (2006). Systematic Control of an Electropneumatic System: Integrator Backstepping and Sliding Mode Control, IEEE Transactions on Control Systems Technology 14(5), 905-913. DOI: 10.1109/TCST.2006.880183

Vörös, J. (2014). Identification of Nonlinear Dynamic Systems with Input Saturation and Output Backlash Using Three-Block Cascade Models, Journal of the Franklin Institute, 351(12), 5455-5466. DOI: 10.1007/s11071-014-1804-4

Wang, J., Sano, A., Chen, T. \& Huang, B. (2009). Identification of Hammerstein Systems without Explicit Parameterisation of Non-Linearity, International Journal of Control, 82(5), 937-952. DOI: $10.1080 / 00207170802382376$

Zhang, Y. M., Chamseddine, A. Rabbath, C. A. Gordon, B. W. Su, C. Y. Rakheja, S. Fulford, C. Apkarian, J. \& Gosselin, P. (2013). Development of Advanced FDD and FTC Techniques with Application to an Unmanned Quadrotor Helicopter Testbed, Journal of the Franklin Institute, 350(9), 2396-2422. DOI: 10.1016/j.jfranklin. 2013.01.009 